CLINICAL STUDY

\title{
Parathyroidectomy eliminates arrhythmic risk in primary hyperparathyroidism, as evaluated by exercise test
}

Jessica Pepe ${ }^{1}$, Mario Curione ${ }^{1}$, Sergio Morelli ${ }^{1}$, Marisa Varrenti ${ }^{1}$, Camillo Cammarota ${ }^{2}$, Mirella Cilli ${ }^{1}$, Sara Piemonte ${ }^{1}$, Cristiana Cipriani ${ }^{1}$, Claudio Savoriti ${ }^{1}$, Orlando Raimo ${ }^{1}$, Federica De Lucia ${ }^{1}$, Luciano Colangelo ${ }^{1}$, Carolina Clementelli ${ }^{1}$, Elisabetta Romagnoli ${ }^{1}$ and Salvatore Minisola ${ }^{1}$

Departments of ${ }^{1}$ Internal Medicine and Medical Disciplines and ${ }^{2}$ Mathematics, 'Sapienza' University of Rome, Viale del Policlinico 155, O0161 Rome, Italy

(Correspondence should be addressed to J Pepe; Email: jesspepe@tin.it)

\begin{abstract}
Objective: To investigate whether parathyroidectomy (PTx) reverses risk factors for arrhythmias related to the QT dynamic changes evaluated during bicycle ergometry exercise test (ET).

Methods: Twenty-four postmenopausal women with primary hyperparathyroidism (PHPT) (mean age $60.0 \pm 8.4$ years) and 30 sex- and age-matched controls underwent ET, echocardiography, and biochemical evaluation. The following stages were considered during ET: rest, peak exercise, and recovery. The patients were randomized to two groups: 12 underwent PTx (group A) and 12 were followed-up conservatively (group B). After 6 months, the patients were studied again.

Results: Groups A and B showed no differences in mean baseline biochemical values, echocardiographic parameters, and QTc interval. PHPT patients showed an increased occurrence of ventricular premature beats (VPBs) during ET compared with controls (37.0 vs $6.6 \%, P=0.03)$. Serum calcium level was a predictor of VPBs $(P=0.05)$. Mean value of QTc was in the normal range at baseline (group A: $401 \pm 16.9$; group B: $402.25 \pm 13.5 \mathrm{~ms})$ but significantly lower than controls $(417.8 \pm 25.1 \mathrm{~ms}$, $P<0.01)$. A negative correlation was found between $Q T c$ and calcium values $(P=0.03)$. Physiological reduction of QTc interval from rest to peak exercise was not observed in PHPT patients before surgery. After PTx, group A had a significant reduction in VPBs compared with baseline (at baseline, 5 of 12 vs none of 12 patients after PTx, $P=0.03$ ) and a restored normal QT adaptation during ET. Group B showed no significant changes after a 6 -month period.

Conclusions: PTx reduces the occurrence of VPBs and restored the QTc adaptation during ET.
\end{abstract}

European Journal of Endocrinology 169 255-261

\section{Introduction}

Convincing evidence has accumulated, indicating that patients with primary hyperparathyroidism (PHPT) may have an increased risk of death, mainly as a consequence of cardiovascular complications, even though the data on those patients with asymptomatic PHPT are limited and controversial $(1,2)$. In this context, it is not known whether arrhythmias play any role in cardiovascular mortality in PHPT patients. We previously reported an increased risk of arrhythmias in PHPT patients evaluated by bicycle ergometry exercise test (ET) (3). This myocardial electrical vulnerability is probably related to hypercalcemia, which is the main biochemical feature of PHPT together with unsuppressed levels of parathyroid hormone (PTH) (4).

A recent paper showed the involvement of ionized calcium-related function in the principal mechanism underlying arrhythmias such as focal ectopic activity due to after depolarization and re-entrant mechanism (5). Serum calcium levels are also negatively associated with QT interval duration both in PHPT patients and in normal subjects $(6,7,8)$. Indeed, we previously reported a relatively short $\mathrm{QT}$ interval and its different adaptation during ET in PHPT compared with normal controls (3). It is well known that shortening of QT interval duration is associated with increased risk of arrhythmias and sudden cardiac death (9); furthermore and more importantly, it has been recently shown that QT interval duration was associated with increased mortality risk, even within the normal reference range in the general population (10).

Therefore, it is of interest to know whether successful parathyroidectomy (PTx), by reversing the concomitant biochemical abnormalities, potentially reverses or even inhibits the progression of cardiovascular abnormalities that negatively affect the prognosis of these patients. Several studies reported a substantial improvement after PTx in the metabolic and the cardiovascular risk factors, also measured by echocardiogram, while other studies do not support this finding $(11,12,13,14,15$, $16,17,18,19,20,21)$. Interestingly, there are no 
studies regarding the effects of PTx on arrhythmic risk, in PHPT patients, evaluated during bicycle ergometry ET.

The aim of this study was to investigate whether PTx can eliminate risk factors for the occurrence of arrhythmias related to QT dynamic changes evaluated by ET.

\section{Subjects and methods}

Thirty-seven consecutive Caucasian postmenopausal women (age range 45-75 years) diagnosed as having PHPT at the Mineral Metabolism Centre of the University of Rome 'Sapienza' (Italy), between November 2009 and April 2011, were enrolled. The diagnosis of PHPT was made following the finding of hypercalcemia with an inappropriately high plasma level of PTH. During the same period, in the same center, 30 Caucasian healthy volunteer age-matched postmenopausal women were enrolled as a control group. Exclusion criteria were as follows: absolute contraindications to exercise stress test or uninterpretable ECG (left bundle branch block, presence of pacemaker, and pre-excitation), hypertrophic cardiomyopathy, and severe valvular disease. None of the patients enrolled were treated with drugs that could interfere with the QT interval. Other exclusion criteria were known coronary artery disease, diabetes mellitus type 1 and type 2, hypothyroidism and hyperthyroidism, hypo-hyperkalemia, hypo-hypermagnesemia, creatinine clearance $(\mathrm{CrCl})<60 \mathrm{ml} / \mathrm{min}$, and BMI $>30 \mathrm{~kg} / \mathrm{m}^{2}$. All patients gave written informed consent. Seven patients affected by PHPT were excluded due to the presence of co-morbidities. The study was approved by our local ethics committee.

Mineral metabolism was evaluated by measuring total $\mathrm{Ca}$, ionized calcium $\left(\mathrm{Ca}^{++}\right)$, phosphorus $(\mathrm{P})$, creatinine $(\mathrm{Cr}), 25-\mathrm{OH}$-vitamin D (25(OH)D), and PTH, as described previously $(22,23,24)$. $\mathrm{CrCl}$ was calculated using the formula of Cockcroft \& Gault (25). TSH was measured using RIA (IRMA TSH-US, Irma ct, Radim Diagnostic, Pomezia, Italy) (26).

In order to exclude patients with contraindications to ET, a transthoracic echocardiography was performed using a Toshiba Aplio CV instrument. The recordings were made with patients recumbent in the left semilateral position. Left ventricular end-diastolic diameter and left ventricular end-systolic diameter, interventricular septum thickness, posterior wall thickness, and transverse diameter of left atrium (LA) were measured according to standards recommended by the American Society of Echocardiography (27). The ejection fraction (EF \%) was calculated by Simpson's rule in apical fourchamber and two-chamber views. The velocities of early transmitral diastolic flow (E) and flow velocity during atrial contraction (A) and its ratio (E/A) were also measured.

Each patient underwent a symptom-limited upright bicycle ergometry ET (Medtronic M 700 Dynavit PPG Hellige), according to modified Bruce's protocol. All subjects reached $85 \%$ of their age-predicted maximal heart rates (28). Blood pressure was monitored during exercise and recovery every 2 min; 12-lead ECG was acquired during exercise and recovery with PC ECG Norav 1200 Medical Ltd software, Yokneam, Israel. QT intervals were measured via standard 12-lead ECG at rest readings. During ET, QT intervals were measured in V5 lead in order to exclude artifacts. We chose QT from lead $\mathrm{V} 5$, according to a previous study (29), because the T wave in this lead was often the tallest and most evident; it was beyond the purpose of this study to discriminate whether the results differed according to the lead selected for QT measurement. All the QT intervals were adjusted using Bazett correction formula $(\mathrm{QTc}=\mathrm{QT} / \sqrt{\mathrm{RR}})(30)$. The data including heart rate, blood pressure, and QTc interval are reported for the following stages: rest, peak exercise, and recovery. The recovery stage included the first stage ( 2 min after peak exercise) and the fifth stage (10 min after peak exercise). In previous studies, the heart rate recovery, the first few minutes after peak exercise, was shown to be a predictor of mortality (31, 32). Thus, early and late recovery after adrenergic stimulation at peak exercise was evaluated.

Following initial assessment, the remaining 30 patients were randomized to either PTx or conservative management; randomization blocks comprised six patients each. However, six patients (three in each group) did not accept this randomization strategy because they wanted to have immediate neck exploration; therefore, they abandoned the study. Six months following the initial evaluation, both groups of patients, those who were randomized to surgery and those who were followed conservatively, underwent the same biochemical evaluations, cardiovascular procedures,

Table 1 Baseline anthropometric parameters and biochemical values in PHPT patients who underwent parathyroidectomy (group A), in those who did not undergo surgery (group B) and controls. Results are presented as mean \pm 1 s.D.

\begin{tabular}{lccc}
\hline & $\begin{array}{c}\text { Group A } \\
(n=12)\end{array}$ & $\begin{array}{c}\text { Group B } \\
(n=12)\end{array}$ & $\begin{array}{c}\text { Controls } \\
(n=30)\end{array}$ \\
\hline Age (years) & $59.8 \pm 7.2$ & $60.15 \pm 9.0$ & $59.2 \pm 6.2$ \\
Years since meno- & $9.4 \pm 6.6$ & $14.2 \pm 11.9$ & $10.7 \pm 13.1$ \\
$\quad$ pause (years) & & & \\
BMl (kg/m $\left.{ }^{2}\right)$ & $24.1 \pm 4.7$ & $26.2 \pm 4.2$ & $24.6 \pm 2.9$ \\
Creatinine clear- & $87.7 \pm 24.9$ & $83.6 \pm 16.0$ & $86.2 \pm 26.5$ \\
$\quad \begin{array}{l}\text { ance (ml/min } \\
\left.\quad 1.73 \mathrm{~m}^{2}\right)\end{array}$ & & & \\
Calcium (mmol/l) & $2.7 \pm 0.1$ & $2.7 \pm 0.1$ & $2.30 \pm 0.14^{*, \dagger}$ \\
lonized calcium & $1.45 \pm 0.16$ & $1.39 \pm 0.03$ & $1.22 \pm 0.04^{*, \dagger}$ \\
$\quad(\mathrm{mmol} / \mathrm{l})$ & & & \\
Phosphorus & $0.89 \pm 0.11$ & $0.92 \pm 0.12$ & $1.25 \pm 0.15^{*, \dagger}$ \\
$\quad(\mathrm{mmol} / \mathrm{l})$ & & & \\
$25(\mathrm{OH}) \mathrm{D}$ (nmol/l) & $25.1 \pm 8.2$ & $27.5 \pm 4.7$ & $22.5 \pm 10.5$ \\
$\mathrm{PTH}(\mathrm{ng} / \mathrm{l})$ & $80.3 \pm 23.5$ & $83.4 \pm 23.1$ & $34.2 \pm 10.3^{*, \dagger}$ \\
TSH (mlU/l) & $1.4 \pm 0.3$ & $1.7 \pm 0.5$ & $1.7 \pm 1.4$ \\
\hline
\end{tabular}

PTH, parathyroid hormone; $25(\mathrm{OH}) \mathrm{D}, 25(\mathrm{OH})$ vitamin $\mathrm{D},{ }^{*} P \leq 0.01$ group $A$ vs controls and ${ }^{\dagger} P \leq 0.01$ group $\mathrm{B}$ vs controls. 
Table 2 Basal echocardiographic parameters in PHPT patients who underwent parathyroidectomy (group A), in those who did not undergo surgery (group B) and in control subjects. Results are presented as mean \pm 1s.D.

\begin{tabular}{|c|c|c|c|c|c|}
\hline & \multicolumn{2}{|c|}{ Group A $(n=12)$} & \multicolumn{2}{|c|}{ Group B $(n=12)$} & \multirow[b]{2}{*}{ Controls $(n=30)$} \\
\hline & Baseline & After 6 months & Baseline & After 6 months & \\
\hline EF (\%) & $63.85 \pm 5.3$ & $63.75 \pm 4.4$ & $65.5 \pm 4.6$ & $66.1 \pm 4.2$ & $66.5 \pm 5.0$ \\
\hline IVS (mm) & $10.0 \pm 0.85$ & $10.3 \pm 1.8$ & $10.1 \pm 1.1$ & $10.2 \pm 1.9$ & $9.8 \pm 1.5$ \\
\hline PWT (mm) & $8.7 \pm 0.8$ & $8.8 \pm 1.5$ & $8.8 \pm 1.2$ & $8.2 \pm 1.5$ & $9.1 \pm 1.3$ \\
\hline$E / A$ & $0.9 \pm 0.3$ & $0.9 \pm 0.3$ & $0.9 \pm 0.2$ & $1.1 \pm 0.35$ & $1.0 \pm 0.25$ \\
\hline $\mathrm{LA}(\mathrm{mm})$ & $36.3+1.7$ & $33.0+93.0$ & $35.6+2.4$ & $33.5+3.7$ & $34.6+2.8$ \\
\hline LVDD (mm) & $44.7 \pm 3.1$ & $43.6 \pm 4.1$ & $42.3 \pm 4.42$ & $44.2 \pm 5.05$ & $47.7 \pm 13.7$ \\
\hline LVDS (mm) & $28.3 \pm 3.1$ & $21.2 \pm 13.35$ & $25.8 \pm 3.7$ & $23.4 \pm 8.9$ & $25.9 \pm 4.1$ \\
\hline
\end{tabular}

EF, ejection fraction; IVS, interventricular septum thickness; PWT, posterior wall thickness; E/A, velocities of early transmitral diastolic flow (E) and flow velocity during atrial contraction (A); LA, transverse diameter of left atrium; LVEDD, left ventricular end-diastolic diameter; LVESD, left ventricular end-systolic diameter.

symptom-limited upright bicycle ergometry ET, and echocardiogram.

\section{Statistical analysis}

Statistical analysis was performed using SPSS for Windows version 13 (SPSS, Inc.). Categorical variables are described with numbers and percentages. Continuous variables are expressed as mean value \pm s.e.m. Because the data distribution did not follow a Gaussian curve, nonparametric tests were carried out. Variations between the various times considered were tested using the Friedman test and the Wilcoxon test. Differences between groups were assessed with $\chi^{2}$ test for categorical variables. A $P$ value $<0.05$ was considered statistically significant.

\section{Results}

As a whole, the PHPT group (mean years from biochemical diagnosis: $2.3 \pm 1.9)$ included three patients with mild PHPT (patients with serum calcium levels not higher than $1 \mathrm{mg} / \mathrm{dl}$ with respect to the normal range, or evidence of bone, renal, or stone disease) (33). In the PHPT group, four subjects were under 50 years. Except for three patients with mild PHPT, the remaining sample was symptomatic and met current indications for surgery (osteoporosis was diagnosed in 70\% of patients and kidney stones in 38\% of patients) (33). There were no differences concerning traditional cardiovascular risk factors between patients and controls (hypertension was diagnosed in 50 vs $24 \%$ of subjects, dyslipidemia in 11 vs $14 \%$ of subjects, and a positive familial history for cardiovascular disease in 10 vs $14 \%$ of subjects). The diagnosis of dyslipidemia was made on the basis of anamnestic data because we did not measure cholesterol level.

After randomization, 12 patients underwent PTx (group A, $n=12$ ) within 15 days after enrollment in the study. The remaining samples were followed conservatively (group $\mathrm{B}, n=12$ ). The cardiovascular risk factors considered so far did not differ between groups $\mathrm{A}$ and $\mathrm{B}$.

Anthropometric and biochemical values of the patients are shown in Table 1. There was no significant difference between PHPT patients and controls, apart from the expected mean higher levels of total, ionized calcium and PTH and mean lower levels of phosphorus in PHPT patients. The echocardiographic parameters of

Table 3 Systolic blood pressure (BP s), diastolic blood pressure (BP d), and heart rate (HR) during each stage of exercise test in PHPT patients and controls. Results are presented as mean \pm 1s.D.

\begin{tabular}{|c|c|c|c|c|c|}
\hline & \multicolumn{2}{|c|}{ Group A $(n=12)$} & \multicolumn{2}{|c|}{ Group B $(n=12)$} & \multirow[b]{2}{*}{ Controls $(n=30)$} \\
\hline & Baseline & After 6 months & Baseline & After 6 months & \\
\hline $\mathrm{BP} s \mathrm{R}$ & $119.5 \pm 12.3$ & $126.8 \pm 10.3$ & $125.45 \pm 14.4$ & $129.2 \pm 10.6$ & $123.8 \pm 15.3$ \\
\hline $\mathrm{BP} d \mathrm{R}$ & $75 \pm 8.7$ & $76.4 \pm 9.0$ & $78.2 \pm 5.7$ & $78.75 \pm 7.4$ & $80.3 \pm 11.2$ \\
\hline HR R & $86.8 \pm 9.4$ & $88.9 \pm 11.5$ & $89.2 \pm 14.4$ & $86.1 \pm 13.6$ & $88.2 \pm 11.6$ \\
\hline BP S P & $160.4 \pm 11.7$ & $172.7 \pm 17.9$ & $171.8 \pm 20.8$ & $168.7 \pm 8.5$ & $171.9 \pm 18.5$ \\
\hline $\mathrm{BP} d \mathrm{P}$ & $93.2 \pm 7.8$ & $97.7 \pm 12.5$ & $95.45 \pm 9.9$ & $100.4 \pm 11.0$ & $95.8 \pm 13.6$ \\
\hline $\mathrm{HR}$ & $138.6 \pm 8.4$ & $130.0 \pm 12.6$ & $142.9 \pm 17.7$ & $127.75 \pm 17.7$ & $135.2 \pm 15.7$ \\
\hline BP s 1st Rc & $144.5 \pm 13.7$ & $149.0 \pm 15.4$ & $153.2 \pm 14.5$ & $154.7 \pm 9.25$ & $153.2 \pm 18.6$ \\
\hline $\mathrm{BP} d 1 \mathrm{st} \mathrm{Rc}$ & $77.3 \pm 12.7$ & $84.5 \pm 4.9$ & $84.1 \pm 8.7$ & $85.0 \pm 5.4$ & $80.0 \pm 11.8$ \\
\hline HR 1st Rc & $92.3 \pm 11.2$ & $88.4 \pm 8.7$ & $99.7 \pm 20.7$ & $90.3 \pm 12.9$ & $96.51 \pm 13.8$ \\
\hline BP $s$ 5th Rc & $110.9 \pm 9.4$ & $116.0 \pm 4.5$ & $114.1 \pm 13.6$ & $117.5 \pm 6.2$ & $115.0 \pm 16.1$ \\
\hline BP d 5th Rc & $72.7 \pm 7.9$ & $78.0 \pm 5.2$ & $75.9 \pm 9.7$ & $77.7 \pm 6.3$ & $74.3 \pm 12.4$ \\
\hline HR 5th R & $83.2 \pm 10.5$ & $84.1 \pm 11.3$ & $86.45 \pm 13.1$ & $82.05 \pm 9.1$ & $84.8 \pm 11.7$ \\
\hline
\end{tabular}

$\mathrm{BP}$, blood pressure; HR, heart rate; R, rest; $\mathrm{P}$, peak exercise; 1st Rc, 1st stage of recovery; 5th Rc, 5th stage of recovery. 
Table 4 Length of QTc interval (ms) in V5 lead during every stage of exercise test in PHPT patient group A and group B, and control subjects. Results are presented as mean \pm 1 s.D.

\begin{tabular}{|c|c|c|c|c|c|}
\hline & \multicolumn{2}{|c|}{ Group A $(n=12)$} & \multicolumn{2}{|c|}{ Group B $(n=12)$} & \multirow[b]{2}{*}{ Controls $(n=30)$} \\
\hline & Baseline & After 6 months & Baseline & After 6 months & \\
\hline $\begin{array}{l}\text { QTc at rest } \\
\text { QTc at peak exercise } \\
\text { QTc at first stage of } \\
\text { recovery }\end{array}$ & $\begin{array}{l}401.0 \pm 16.9 \\
396.6 \pm 18.2 \\
399.6 \pm 15.7\end{array}$ & $\begin{array}{l}420.9 \pm 10.1^{\dagger} \\
397.7 \pm 8.8 \\
406.3 \pm 15.1\end{array}$ & $\begin{array}{c}402.25 \pm 13.5 \\
396.5 \pm 27.8 \\
393.2 \pm 15.35\end{array}$ & $\begin{array}{l}401.1 \pm 17.7^{\|} \\
398.0 \pm 12.8 \\
394.2 \pm 18.45\end{array}$ & $\begin{array}{c}417.8 \pm 25.1^{\star, \ddagger, \S, \|} \\
403.38 \pm 28.6 \\
405.5 \pm 16.5^{\star, \ddagger, \S, \|}\end{array}$ \\
\hline $\begin{array}{l}\text { QTc at fifth stage of } \\
\text { recovery }\end{array}$ & $400.9 \pm 15.8$ & $413.4 \pm 18.15$ & $401.5 \pm 15.7$ & $396.4 \pm 17.0^{\| \prime}$ & $414.5 \pm 21.2^{*, \ddagger}$ \\
\hline
\end{tabular}

Group $A^{*} P \leq 0.01$ baseline vs controls, group $A^{\dagger} P \leq 0.01$ baseline vs after 6 months, group $B^{\ddagger} P \leq 0.01$ baseline vs controls, group $B^{\S} P \leq 0.01$ after 12 months vs controls. " $P \leq 0.05$ group A after 6 months vs group B after 6 months.

PHPT patients and controls are given in Table 2. These indices were within normal values and did not differ either between PHPT patients of groups A and B or when compared with controls. Table 3 shows mean blood pressure and heart rate values for every stage of the ET considered; there were no significant differences between the two groups of PHPT patients when compared with controls.

At rest, mean values of QTc interval in PHPT patients were significantly lower compared with those found in controls, even though they were within the normal range. There was no difference between QTc values in every ET stage in groups A and B (Table 4). PR and QRS measurements were also normal and their mean values did not differ between PHPT patients and controls (data not shown). As expected, the QTc values at rest showed a negative correlation with serum total calcium levels $(R=-0.30, P=0.03)$ as shown in Fig. 1 .

The ETs were negative for ischemia-induced symptoms or signs, both in patients and in controls. However, an increased occurrence of ventricular premature beats (VPBs) in PHPT patients during ET was observed at baseline compared with controls $(37.0$ vs $6.6 \%$, $P=0.01)$. VPBs were predicted by total calcium levels by regression analysis $\left(R^{2}=0.17, \beta=-0.41, P=0.05\right)$ but not by QTc at rest. QTc at rest was a predictor of QTc in every stage, as we previously demonstrated (3).

The difference in the dynamic changes of the QTc interval between the PHPT and controls is shown in Fig. 2 (group A) and Fig. 3 (group B). Contrary to what has been observed in the control subjects, the physiological reduction of QTc interval from rest to peak exercise was not seen in groups A and B of PHPT patients. In fact, considering the delta QTc interval, that is, the difference between every stage of the ET in both groups, only delta QTc rest-QTc peak exercise showed statistically significant differences between PHPT patients and controls (group A vs controls: $-1 \pm 1$ vs $-13.8 \pm 18$, group B vs controls: $-0.1 \pm 2$ vs $-13.8 \pm$ 18 , both $P=0.01$ ). There was no difference at baseline between groups A and B considering QTc at every stage and delta QTc.

After 6 months, patients who underwent PTx (group A) showed a significant reduction of mean ionized calcium levels and PTH levels compared with baseline $(1.45 \pm 0.16$ vs $1.22 \pm 0.03 \mathrm{mmol} / \mathrm{l}, 80.3 \pm 23.55$ vs $34.6 \pm 12.3 \mathrm{pg} / \mathrm{ml}$ respectively, all $P<0.001$ ), whereas in group $\mathrm{B}$, these parameters remained stable. The echocardiographic parameters, as well as mean blood pressure and heart rate, did not show any differences after 6 months compared with the baseline, both in groups A and B (Tables 2 and 3).

After 6 months, mean values of QTc at rest in group A were significantly higher compared with mean values found at baseline, although within the normal range; the mean QTc values in group B remained significantly lower compared with controls (Table 4). Figure 2 shows that, after PTx, the dynamic changes in QTc of group A differed compared with the baseline being equal to the controls. In fact, mean values of delta QTc rest-QTc peak exercise did not differ between the parathyroidectomized patients and controls. A significant reduction of VPBs was also observed after PTx (at baseline, 5 of 12 vs none of 12 patients after PTx, $P=0.03)$. Taking into consideration patients in group B (i.e. those who did not undergo PTx), the dynamic changes in QTc remained stable after 6 months and therefore always different from the controls as shown in Fig. 3. There was no significant reduction of VPBs after 6 months ( 4 of 12 patients at baseline vs 5 of 12 patients after 6 months).

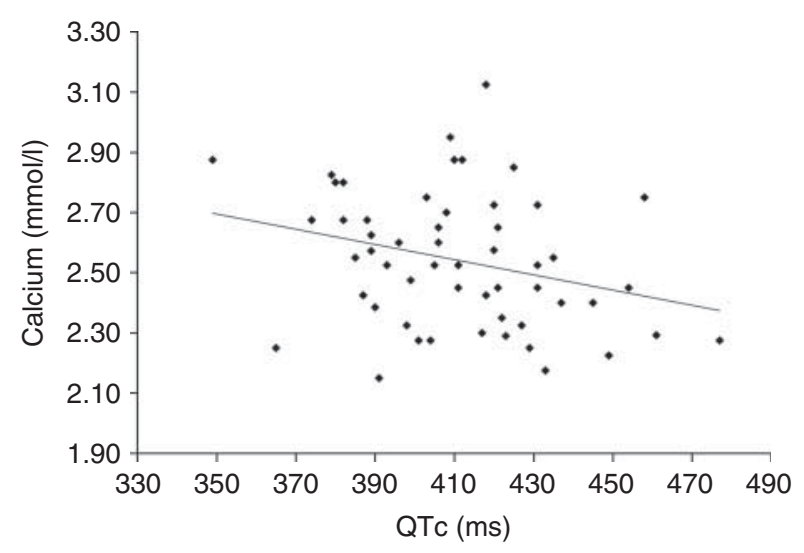

Figure 1 Correlation between calcium and QTc interval at rest in whole sample. 


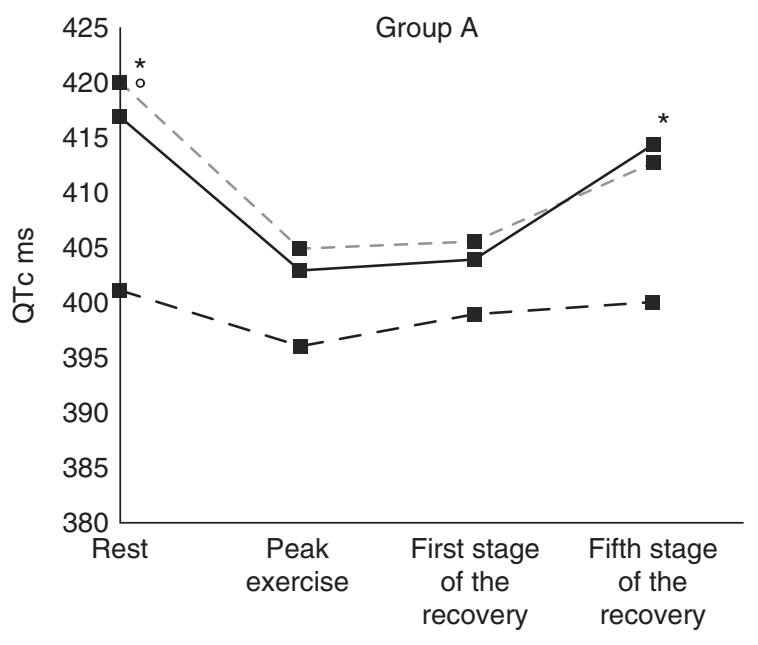

Stages of exercise test

Figure 2 QTc interval during exercise test in PHPT patients (group A) at baseline (dashed black line) and after 6 months since parathyroidectomy (dashed grey line) and controls. ${ }^{\star} P \leq 0.01$, PHPT baseline vs controls (solid black line), ${ }^{\circ} P \leq 0.01$, PHPT baseline vs PHPT after 6 months.

Comparing parathyroidectomized patients after 6 months with those who did not undergo surgery, the first group (group A) showed mean significantly higher QTc values compared with group B at rest and at the fifth stage of recovery during the ET (Table 4). Also mean values of delta QTc rest-QTc peak exercise were significantly different between groups A and B. Moreover, even if the prevalence of VPBs in group A did not differ at the baseline compared with group B, only patients who underwent PTx had a significant reduction of VPBs after 6 months (none in group A vs five patients in group $\mathrm{B}, P=0.03)$.

\section{Discussion}

This is the first randomized study showing a reduction of VPBs and restored physiological QT dynamics, as evaluated by ET, after PTx in patients affected by PHPT. To the best of our knowledge, there is only one study evaluating the effect of PTx on VPBs; even though the authors did not find any changes in the prevalence of VPBs, it is worthy to underline that the investigators enrolled patients with coronary artery disease and diabetes that are well-known confounding factors (13). Our study was carried out under strict exclusion criteria to avoid this bias and with a major strength of randomization.

VPBs in PHPT patients are predicted by total calcium levels, but the mechanism underlying this electrical abnormality is still unclear. Focal ectopic activity due to after depolarization and re-entrant mechanism are probably involved, both being linked due to hypercalcemia (5).

We observed a prolongation of QTc interval after PTx, even though the values registered were always within the normal range. This is probably due to the effect of surgery on serum calcium levels owing to the observed inverse correlation between serum calcium and QTc interval. The importance of this observation is supported by data from the NHANES III, a large sample representative of the general US population that showed a U-shaped relationship between QT interval and mortality end points (10). Shortened and prolonged QT interval durations, even within reference ranges, were associated with increased risks of total cardiovascular and non-cardiovascular deaths (10). Our study also showed restored physiological QTc dynamics during ET after PTx. As can be seen in Fig. 1, the QTc dynamics of parathyroidectomized patients is the same as the QT dynamics observed in the control subjects. Our observation is probably related to the longer QTc at rest (but still within the normal range) that was seen after PTx. In fact, QTc during ET is predicted by QTc at rest, as we previously demonstrated (3). Interestingly, patients who did not undergo PTx continued to show different QTc dynamics. QTc dynamics could represent a risk factor for arrhythmias (34) that PTx is able to reverse.

In our study, PTx did not modify echocardiographic parameters as some previous studies have shown; however, in our sample, these parameters were within the normal range and did not differ from controls, whereas in other studies, patients with left ventricular hypertrophy were enrolled $(13,14)$. Also, mean blood pressure and heart rate during ET, before and after PTx, remained within the normal range and did not differ. This finding is in contrast to the only previous study

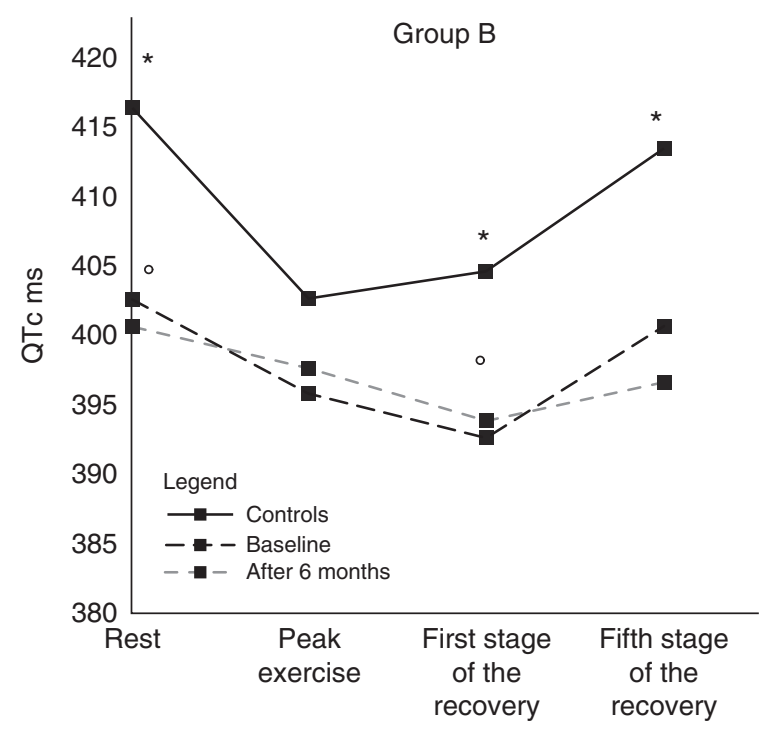

Stages of exercise test

Figure 3 QTc interval during exercise test in PHPT patients (group B) at baseline (dashed black line) and after 6 months (dashed grey line) and controls (solid black line). ${ }^{\star} P \leq 0.01$, PHPT baseline vs controls, ${ }^{\circ} P \leq 0.01$, PHPT baseline vs PHPT after 6 months. 
that investigated ET in PHPT patients, which had many confounding factors (14).

A possible limitation of our study was the relatively small sample size and the exclusion of male subjects from enrollment. Males were excluded because they have a different QTc normal range compared with women. QTc is longer in women, and this would have introduced a bias into our study (35). Therefore, this exclusion strengthens our results. Another limitation of our study was the lack of lipid profile examination since dyslipidemia was diagnosed on the basis of anamnestic data; however, the prevalence of this well-known cardiovascular risk factor did not differ between PHPT patients and controls and between groups A and B.

In conclusion, on the basis of our data, an ET in patients with PHPT could add more information about arrhythmic risk than that provided by the ECG at rest. Therefore, the ET could show arrhythmias occurring during induced tachycardia and could reveal QT dynamics, which if altered represent a risk factor for arrhythmias. We demonstrate that after PTx, there is a significant resolution of cardiovascular abnormalities; indeed, arrhythmias during ET in PHPT patients were no longer observed and QTc dynamics were identical to those observed in normal subjects. The clinical implications of these findings need confirmation with a larger sample and possibly a prolonged follow-up; however, if the results of this investigation are confirmed, our study would likely change the recommendation for PTx that currently focuses only on traditional aspects of PHPT, such as skeletal and renal involvement (33).

\section{Declaration of interest}

The authors declare that there is no conflict of interest that could be perceived as prejudicing the impartiality of the research reported.

\section{Funding}

This research did not receive any specific grant from any funding agency in the public, commercial or not-for-profit sector.

\section{References}

1 Hedbäck G \& Odén A. Increased risk of death from primary hyperparathyroidism an update. European Journal of Clinical Investigation $1998 \quad 4$ 271-276. (doi:10.1046/j.1365-2362. 1998.00289.x)

2 Yu N, Donnan PT, Flynn RW, Murphy MJ, Smith D, Rudman A \& Leese GP. Increased mortality and morbidity in mild primary hyperparathyroid patients. The Parathyroid Epidemiology and Audit Research Study (PEARS). Clinical Endocrinology 201073 30-34.

3 Pepe J, Curione M, Morelli S, Colotto M, Varrenti M, Castro C, D'Angelo A, Cipriani C, Piemonte S, Romagnoli E et al. Arrhythmias in primary hyperparathyroidism evaluated in exercise test. European Journal of Clinical Investigation 201343 208-214. (doi:10.1111/eci.12038)

4 Pepe J, Cipriani C, Pilotto R, De Lucia F, Castro C, Lenge L, Russo S, Guarnieri V, Scillitani A, Carnevale Vet al. Sporadic and hereditary primary hyperparathyroidism. Journal of Endocrinological Investigation 201134 (7 Suppl) 40-44.
5 Nattel S \& Dobrev D. The multidimensional role of calcium in atrial fibrillation pathophysiology: mechanistic insights and therapeutic opportunities. European Heart Journal 201233 1870-1877. (doi:10.1093/eurheartj/ehs079)

6 Lind L \& Ljunghall S. Serum calcium and the ECG in patients with primary hyperparathyroidism. Journal of Electrocardiology 199427 99-103. (doi:10.1016/S0022-0736(05)80092-5)

7 Zhang Y, Post WS, Dalal D, Bansal S, Blasco-Colmenares E, Jan De Beur S, Alonso A, Soliman EZ, Whitsel EA, Brugada R et al. Serum 25-hydroxyvitamin D, calcium, phosphorus, and electrocardiographic QT interval duration: findings from NHANES III and ARIC. Journal of Clinical Endocrinology and Metabolism 201196 1873-1882. (doi:10.1210/jc.2010-2969)

8 Saikawa T, Tsumabuki S, Nakagawa M, Takakura T, Tamura M, Maeda T, Ito S \& Ito M. QT intervals as an index of high serum calcium in hypercalcemia. Clinical Cardiology 198811 75-78. (doi:10.1002/clc.4960110205)

9 Napolitano C, Bloise R, Monteforte N \& Priori SG. Sudden cardiac death and genetic ion channelopathies: long QT, Brugada, short QT, catecholaminergic polymorphic ventricular tachycardia, and idiopathic ventricular fibrillation. Circulation 2012125 20272034. (doi:10.1161/CIRCULATIONAHA.111.055947)

10 Zhang Y, Post WS, Dalal D, Blasco-Colmenares E, Tomaselli GF \& Guallar E. QT-interval duration and mortality rate: results from the Third National Health and Nutrition Examination Survey. Archives of Internal Medicine 2011171 1727-1733. (doi:10. 1001/archinternmed.2011.433)

11 Näppi S, Saha H, Virtanen V, Limnell V, Sand J, Salmi J \& Pasternack A. Left ventricular structure and function in primary hyperparathyroidism before and after parathyroidectomy. Cardiology 200093 229-233. (doi:10.1159/000007031)

12 Stefenelli T, Abela C, Frank H, Koller-Strametz J, Globits S, BerglerKlein J \& Niederle B. Cardiac abnormalities in patients with primary hyperparathyroidism: implications for follow-up. Journal of Clinical Endocrinology and Metabolism 199782 106112. (doi:10.1210/jc.82.1.106)

13 Piovesan A, Molineri N, Casasso F, Emmolo I, Ugliengo G, Cesario F \& Borretta G. Left ventricular hypertrophy in primary hyperparathyroidism. Effects of successful parathyroidectomy. Clinical Endocrinology 199950 321-328. (doi:10.1046/j.1365-2265. 1999.00651.x)

14 Nilsson IL, Aberg J, Rastad J \& Lind L. Left ventricular systolic and diastolic function and exercise testing in primary hyperparathyroidism - effects of parathyroidectomy. Surgery 2000128 895902. (doi:10.1067/msy.2000.110240)

15 Curione M, Amato S, Di Bona S, Petramala L, Cotesta D \& Letizia C. Parathyroidectomy erase increased myocardial electrical vulnerability in patients with primary hyperparathyroidism. International Journal of Cardiology 2010141 201-202. (doi:10.1016/j.ijcard. 2008.11.067)

16 Curione M, Letizia C, Amato S, Di Bona S, Di Fazio F, Minisola S, Mazzuoli G \& D'Erasmo E. Increased risk of cardiac death in primary hyperparathyroidism: what is a role of electrical instability? International Journal of Cardiology 2007121 200202. (doi:10.1016/j.ijcard.2006.08.072)

17 Vestergaard P, Mollerup CL, Frøkjaer VG, Christiansen P, BlichertToft M \& Mosekilde L. Cardiovascular events before and after surgery for primary hyperparathyroidism. World Journal of Surgery 200327 216-222. (doi:10.1007/s00268-002-6589-9)

18 Walker MD, Rundek T, Homma S, Ditullio M, Iwata S, Lee JA, Choi J, Liu R, Zhang C, McMahon DJ et al. Effect of parathyroidectomy on subclinical cardiovascular disease in mild primary hyperparathyroidism. European Journal of Endocrinology 2012167 277-285.

19 Osto E, Fallo F, Pelizzo MR, Maddalozzo A, Sorgato N, Corbetti F, Montisci R, Famoso G, Bellu R, Lüscher TF et al. Coronary microvascular dysfunction induced by primary hyperparathyroidism is restored after parathyroidectomy. Circulation $2012 \mathbf{1 2 6}$ 1031-1039. (doi:10.1161/CIRCULATIONAHA.111.081307)

20 Bollerslev J, Rosen T, Mollerup CL, Nordenström J, Baranowski M, Franco C, Pernow Y, Isaksen GA, Godang K, Ueland T et al. Effect of 
surgery on cardiovascular risk factors in mild primary hyperparathyroidism. Journal of Clinical Endocrinology and Metabolism 2009 94 2255-2261. (doi:10.1210/jc.2008-2742)

21 Ishay A, Herer P \& Luboshitzky R. Effects of successful parathyroidectomy on metabolic cardiovascular risk factors in patients with severe primary hyperparathyroidism. Endocrine Practice 201117 584-590. (doi:10.4158/EP10321.OR)

22 Pepe J, Petrucci MT, Nofroni I, Fassino V, Diacinti D, Romagnoli E \& Minisola S. Lumbar bone mineral density as the major factor determining increased prevalence of vertebral fractures in monoclonal gammopathy of undetermined significance. British Journal of Haematology $2006 \mathbf{1 3 4} 485-490$. (doi:10.1111/j.13652141.2006.06217.x)

23 Maggio D, Cherubini A, Lauretani F, Russo RC, Bartali B, Piereandrei M, Ruggiero C, Macchiarulo MC, Giorgino R, Minisola $\mathrm{S}$ et al. 25(OH)D serum levels decline with age earlier in women than in men and less efficiently prevent compensatory hyperparathyroidism in older adults. Journals of Gerontology. Series A, Biological Sciences and Medical Sciences $2005601414-1419$. (doi:10.1093/gerona/60.11.1414)

24 Carnevale V, Dionisi S, Nofroni I, Romagnoli E, Paglia F, De Geronimo S, Pepe J, Clemente G, Tonnarini G \& Minisola S. Potential clinical utility of a new IRMA for parathyroid hormone in postmenopausal patients with primary hyperparathyroidism. Clinical Chemistry 200450 626-631. (doi:10.1373/clinchem. 2003.026328)

25 Cockcroft DW \& Gault MH. Prediction of creatinine clearance from serum creatinine. Nephron 197616 31-41. (doi:10.1159/ 000180580)

26 Caldwell G, Kellett HA, Gow SM, Beckett GJ, Sweeting VM, Seth J \& Toft AD. A new strategy for thyroid function testing. Lancet 19851 1117-1119. (doi:10.1016/S0140-6736(85)92429-8)

27 Lang RM, Bierig M, Devereux RB, Flachskampf FA, Foster E, Pellikka PA, Picard MH, Roman MJ, Seward J, Shanewise JS et al. Recommendations for chamber quantification: a report from the American Society of Echocardiography's Guidelines and Standards Committee and the Chamber Quantification Writing Group, developed in conjunction with the European Association of Echocardiography, a branch of the European Society of Cardiology. Journal of the American Society of Echocardiography 200518 14401463. (doi:10.1016/j.echo.2005.10.005)

28 Gibbons RJ, Balady GJ, Bricker JT, Chaitman BR, Fletcher GF, Froelicher VF, Mark DB, McCallister BD, Mooss AN, O'Reilly MG et al.
ACC/AHA 2002 guideline update for exercise testing: summary article: a report of the American College of Cardiology/American Heart Association Task Force on Practice Guidelines (Committee to Update the 1997 Exercise Testing Guidelines). Circulation 2002106 1883-1892. (doi:10.1161/01.CIR.0000034670. $06526.15)$

29 Malfatto G, Zaza A \& Facchini M. Different effects of antiarrhythmic drugs on the rate-dependency of QT interval: a study with amiodarone and flecainide. Journal of Cardiovascular Pharmacology 200750 535-540. (doi:10.1097/FJC.0b013e3181451473)

30 Bazzet HD. An analysis of the time relations of electrocardiograms. Heart 19207 353-370.

31 Nishime EO, Cole CR, Blackstone EH, Pashkow FJ \& Lauer MS. Heart rate recovery and treadmill exercise score as predictors of mortality in patients referred for exercise ECG. Journal of the American Medical Association 2000284 1392-1398. (doi:10. 1001/jama.284.11.1392)

32 Cole CR, Blackstone EH, Pashkow FJ, Snader CE \& Lauer MS. Heart-rate recovery immediately after exercise as a predictor of mortality. New England Journal of Medicine 1999341 1351-1357. (doi:10.1056/NEJM199910283411804)

33 Bilezikian JP, Khan AA, Potts JT Jr \& on behalf of the Third International Workshop on the Management of Asymptomatic Primary Hyperthyroidism. Guidelines for the Management of Asymptomatic Primary Hyperparathyroidism: Summary Statement from the Third International Workshop. Journal of Clinical Endocrinology and Metabolism 200994 335-339. (doi:10.1210/ jc.2008-1763)

34 Gill JS, Baszko A, Xia R, Ward DE \& Camm AJ. Dynamics of the QT interval in patients with exercise-induced ventricular tachycardia in normal and abnormal hearts. American Heart Journal 1993126 1357-1363. (doi:10.1016/0002-8703(93)90534-G)

35 Mayuga KA, Parker M, Sukthanker ND, Perlowski A, Schwartz JB \& Kadish AH. Effects of age and gender on the QT response to exercise. American Journal of Cardiology 200187 163-167. (doi:10.1016/S0002-9149(00)01309-6)

Received 6 April 2013

Revised version received 3 June 2013

Accepted 6 June 2013 